\title{
Apprentissage, jeu sérieux et « détournement sérieux de jeu »
}

\section{CHRONIQUE • Intervention éducative}

La présence du jeu à l'école est ancienne et se décline autant sous la forme du jeu sans finalité pédagogique apparente de la récréation, que de l'activité ludique en classe. Cependant, depuis les quarante dernières années, le jeu s'est transformé. Le jeu numérique des années 70 s'est en effet popularisé pour devenir, aujourd'hui, une industrie multimilliardaire. Forme d'art qui a maintenant sa place au musée, le jeu numérique a de multiples finalités, qui relèvent du domaine commercial ainsi que de ceux de l'éducation et de la formation.

Nous proposons, à l'enseignant et au formateur qui désirent faire usage de jeux numériques, deux approches que sont le jeu sérieux et le « détournement sérieux de jeu ».

\section{Définitions}

Le jeu numérique constitue un domaine de recherche relativement jeune en éducation dont l'objet d'étude, le jeu - de par sa complexité grandissante - ne fait pas consensus quand il s'agit de le définir (Esdaile, 2012).

Le jeu sérieux est en effet défini de multiples manières depuis 2002 (Ma, Oliveira, Petersen et Hauge, 2013). Cependant, nous retiendrons qu'il inclut des objectifs pédagogiques clairement définis, qui se manifestent dans un environnement de jeu réaliste ou artificiel (Sauvé, 2008) comportant des règles et des défis. Ainsi, le jeu sérieux est conçu spécifiquement pour l'apprentissage et la formation. Le jeu Slice Fractions [http://ululab.com/fr/slice fractions/] en est un 
bel exemple. Conçu par la firme montréalaise Ululab en collaboration avec des experts en pédagogie de l'UQAM, ce jeu a pour objectif l'apprentissage des fractions dans un univers préhistorique où le joueur aide un petit mammouth à surmonter des obstacles.

Le détournement sérieux de jeu est plutôt défini comme étant l'utilisation du jeu numérique en contexte d'apprentissage. Le jeu n'est pas conçu aux fins de l'apprentissage, mais il peut permettre à l'utilisateur de développer plusieurs compétences et attitudes (Gee, 2007). À titre d'exemple, le jeu de construction Minecraft [https://minecraft.net/], dont le but est d'accumuler des ressources et de construire un environnement tout en se protégeant de nombreux monstres nocturnes, a été utilisé par des milliers d'enseignants à travers le monde pour atteindre différents objectifs pédagogiques.

Ces définitions permettent de constater que le jeu numérique est intéressant dans la mesure où il y a un avantage pédagogique. Peu de gens contesteront cette affirmation. Par contre, comment promouvoir, mesurer et constater les apprentissages résultant de l'utilisation de jeux est beaucoup moins clair. Sur ce point, nous proposons deux avenues qui peuvent être complémentaires, soit tout d'abord l'analyse des données générées par les utilisateurs de jeux sérieux, puis le détournement sérieux qui se manifeste dans l'orientation des finalités d'un jeu existant par les enseignants et les formateurs, et dans la conception de jeux par les étudiants.

\section{Jeu sérieux et valorisation des données sur le joueur}

Toute action dans un environnement numérique, pour le meilleur et pour le pire, laisse des traces. Dans les jeux de nature commerciale, les données servent principalement à améliorer le jeu, puis à définir des profils de joueurs. En éducation et en formation, où les finalités sont tout autres, les données - qui sont également utiles pour l'amélioration des jeux - servent aussi et surtout à valider l'acquisition des compétences (Loh, Sheng et Ifenthaler, 2015). Plus le jeu sérieux et le système qui le diffuse sont complexes, plus grande sera la possibilité d'évaluer un large éventail de compétences. Par exemple, le Carrefour virtuel de jeux éducatifs 2.0 [http://cvje2.savie.ca/], développé par le Centre de recherche public SAVIE en collaboration avec le CEFRIO ${ }^{1}$, permet aux enseignants de créer des questions de différents types qui sont proposées à leurs étudiants sous la forme d'un jeu générique. Un jeu générique est un jeu très connu comme le tic-tac-toe où l'apprenant peut placer un $\mathrm{X}$ ou un $\mathrm{O}$ sur la grille s'il donne une bonne réponse à la question formulée par l'enseignant. À partir d'un jeu générique, celui-ci peut ainsi créer plusieurs questionnaires - autrement dit plusieurs jeux - pouvant intégrer des images, de la vidéo ou de l'audio, et permettant de faire, de manière ludique, une évaluation formative des connaissances de l'apprenant qui doit aussi appliquer une stratégie pour gagner (Plante et Sauvé, 2016).

Le jeu donne des rétroactions lors de la partie et compile un tableau personnalisé des apprentissages, qui identifie les questions ratées et propose des ressources pour l'amélioration de la performance. Pour l'enseignant, un tableau général de gestion donne des pistes sur les questions à reformuler, l'enseignement préalable à améliorer ou l'enseignement ultérieur à préparer. Le jeu sérieux, comme environnement structuré et monitoré, permet ainsi de valoriser des données qui sont autant d'indices sur le parcours d'apprentissage de chaque personne. 


\section{Détournement sérieux et conception de jeu}

Les jeux sérieux comportent certaines limites. Ils sont dispendieux à développer et ne permettent pas facilement d'actualiser des compétences de haut niveau. D'autres solutions sont cependant possibles. L'enseignant peut utiliser un jeu commercial en lui attribuant de nouvelles finalités grâce à la structuration d'un scénario. Par exemple, il peut utiliser des jeux comme Civilization [http://www.civilization.com/] ou Freeciv [http://www.freeciv.org/] pour illustrer les forces en présence, les stratégies et la gestion des ressources lors de la bataille de Québec en 1759.

Un détournement de jeu peut aussi être opéré à un autre niveau, où c'est le concept même du jeu qui est détourné. Le jeu n'est alors plus un moyen d'atteindre ou d'évaluer un objectif pédagogique, comme dans le cas d'un jeu sérieux, mais plutôt l'objectif qui permet de mobiliser, chez l'apprenant, les compétences et les ressources nécessaires à la conception d'un jeu. Il s'agit là d'une transposition de l'apprentissage par l'action (Learning by doing) de Dewey. Il n'y a pas de modalités strictes pour l'enseignant qui désire que les apprenants construisent des jeux. Cependant, il devra par exemple leur fournir des objectifs en termes de contenu disciplinaire à s'approprier et à actualiser dans le jeu, ainsi que les bases techniques d'un logiciel de production de jeux. Plus les apprenants auront de liberté envers les moyens techniques proposés, plus l'enseignant devra maitriser divers logiciels de production de jeux. Il s'agit là, d'une certaine manière, du prix à payer pour faire cheminer les étudiants vers des compétences et des attitudes de haut niveau.

En conclusion, l'utilisation du jeu numérique peut varier selon les moyens financiers, le temps et les connaissances techniques dont dispose l'enseignant. Ainsi, le jeu sérieux et le détournement sérieux de jeu sont deux approches intéressantes à considérer pour favoriser l'apprentissage chez les apprenants.

\section{Références}

Esdaile, M. (2012). Quels rôles pédagogiques pour les jeux numériques? Adjectif. Repéré à http://www.adjectif.net/spip/spip.php?article181

Gee, J. P. (2007). What video games have to teach us about learning and literacy. New York, NY : Palgrave Macmillan.

Loh, C. S., Sheng, Y. et Ifenthaler, D. (2015). Serious games analytics: Theoretical framework. Dans C. S. Loh, Y. Sheng et D. Ifenthaler (dir.), Serious games analytics (p. 3-29). http://dx.doi.org/10.1007/978-3-319-05834-4_1

Ma, M., Oliveira, M. F., Petersen, S. et Hauge, J. B. (dir.). (2013). Serious games development and applications (vol. 8101). Berlin, Heidelberg : Springer Berlin Heidelberg. http://dx.doi.org/10.1007/978-3-642-40790-1

Plante, P. et Sauvé, L. (2016, avril). De joueur à concepteur : design de jeux sérieux pour l'augmentation de l'implication et de la richesse des liens sociaux des personnes aînées. Communication présentée au Digital Intelligence 2016 / Intelligences numériques 2016, Québec, QC. Repéré à http://di2016.org/fileadmin/documents/DI2016 paper_17.pdf

Sauvé, L. (2008, août). Concevoir des jeux éducatifs en ligne : un atout pédagogique pour les enseignants. Communication présentée au Colloque Ludovia, Ax-les-Thermes, Ariège. Repéré à http://www.ludovia.com/tag/louise-sauve/

\section{Note}

1 CEFRIO ou Centre facilitant la recherche et l'innovation dans les organisations, à l'aide des technologies de l'information et de la communication (TIC)

\section{Pour citer cet article}

Plante, P. (2016). Apprentissage, jeu sérieux et «détournement sérieux de jeu». Formation et profession 24(2), $72-74$. http://dx.doi.org/10.18162/fp.2016.a96 